\title{
The value of computer-assisted navigation for bone reconstruction after tumor resection
}

\author{
XUEJUN PENG ${ }^{1}$, FENGPING WANG ${ }^{2}$ and JING SU ${ }^{3}$ \\ Departments of ${ }^{1}$ Traumatology and ${ }^{2}$ Ultrasound, Linyi People's Hospital, Linyi, Shandong 276000; \\ ${ }^{3}$ Community Health Center of Yinque Mountain, Linyi, Shandong 276003, P.R. China
}

Received March 14, 2017; Accepted June 28, 2017

DOI: 10.3892/ol.2017.6523

\begin{abstract}
This study was designed to evaluate the use of computer-assisted navigation with computed tomography (CT) images for bone reconstruction after resection in malignant bone tumor treatment. Forty-five patients with malignant bone tumors were recruited for this study. CT scan images in a computer-assisted navigation system were used to assist during the osteotomy, the pairing with allografts, and the monitoring of the allograft and joint lines to perform joint reconstruction. Our results show that osteotomy and allograft pairing were successful in all patients. The average duration of the osteotomy procedures was $46.8 \pm 12.3 \mathrm{~min}$; and the average pairing time was $32.5 \pm 9.8 \mathrm{~min}$. The anatomical registration points and the three-dimensional virtual CT images were successfully matched. The average error of registration was $0.36 \pm 0.09 \mathrm{~mm}$. Also, the range of tumor resection and allograft osteotomy were successfully paired, with an average error of $0.11 \pm 0.03 \mathrm{~mm}$. No complications such as unequal limbs length or joint deformities occurred after reconstruction. The average follow-up time was $11.6 \pm 3.9$ months. The tumor recurrence rate was $11.1 \%(5 / 45)$ and the survival rate $95.6 \%$ (43/45). The average healing time for the allograft and host bone was $5.5 \pm 1.2$ months and no unexpected internal fixations, fractures or joint collapses occurred. The average

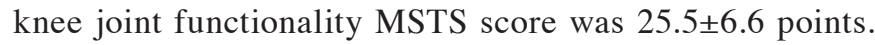
No significant differences were found in the length of tumor resection, rate of negative incision margin, duration of osteotomy or of pairing, registration error or allogeneic bone and defect matching error averages between those patients with tumor recurrence and those without it $(\mathrm{p}>0.05)$. Based on our results, the computer-assisted navigation system for bone reconstruction after malignant tumor resection allows for high precision during osteotomy, delivers a high success rate
\end{abstract}

Correspondence to: Dr Fengping Wang, Department of Ultrasound, Linyi People's Hospital, 27 East Jiefang Road, Linyi, Shandong 276000, P.R. China

E-mail: fod55z@163.com

Key words: computer-assisted navigation, bone tumor, bone reconstruction of pairing, results in great limb function and low complication rates, and is thus a highly successful and safe approach benefiting bone cancer patients.

\section{Introduction}

In the treatment of malignant bone tumors around joints it is essential to perform a complete resection of tumor segments and to strive for the functional reconstruction of the joint (1). Various preoperative assessments such as computed tomography $(\mathrm{CT})$ and magnetic resonance imaging (MRI) are important references for guiding surgical margins (2). Allogeneic joint transplantations and artificial joints are both used in joint functional reconstructions (3). One of the main challenges for surgeons is finding an appropriate replacement for large bone segment defects after tumor resection. The establishment of a digital image inventory based on bone analysis technology and available in a computer-assisted navigation system will aid in matching the necessary surgical resection margins to appropriate allogeneic bone specimens, significantly improving the outcome of such procedures $(4,5)$. Accordingly, this study aims at analyzing the value of a computer-assisted navigation system based on CT images for bone reconstruction after malignant tumor resection in the knee.

\section{Patients and methods}

Participant selection. Forty-five patients diagnosed with malignant bone tumors around the knee joint, at Linyi People's Hospital, were continuously recruited for this study starting October 2014 and lasting until October 2015. All the participants had limb salvage indications, an expected life expectancy longer than 12 months, and accompanying complete clinical data. This study was approved by the Ethics Committee of Linyi People's Hospital. Signed written informed consents were obtained from the patients. All participants signed informed consent forms. In addition, none of the patients enrolled presented knee osteoarthritis, rheumatoid diseases or tumor metastasis. A total of 29 male and 16 female patients participated, their average age was of 52.6 \pm 13.5 years with a range of $38-72 ; 24$ patients had a lesion on the left knee and 21 presented a right knee lesion. In terms of tumor location, 22 cases had a distal femur tumor, and 23 cases a proximal tibia 

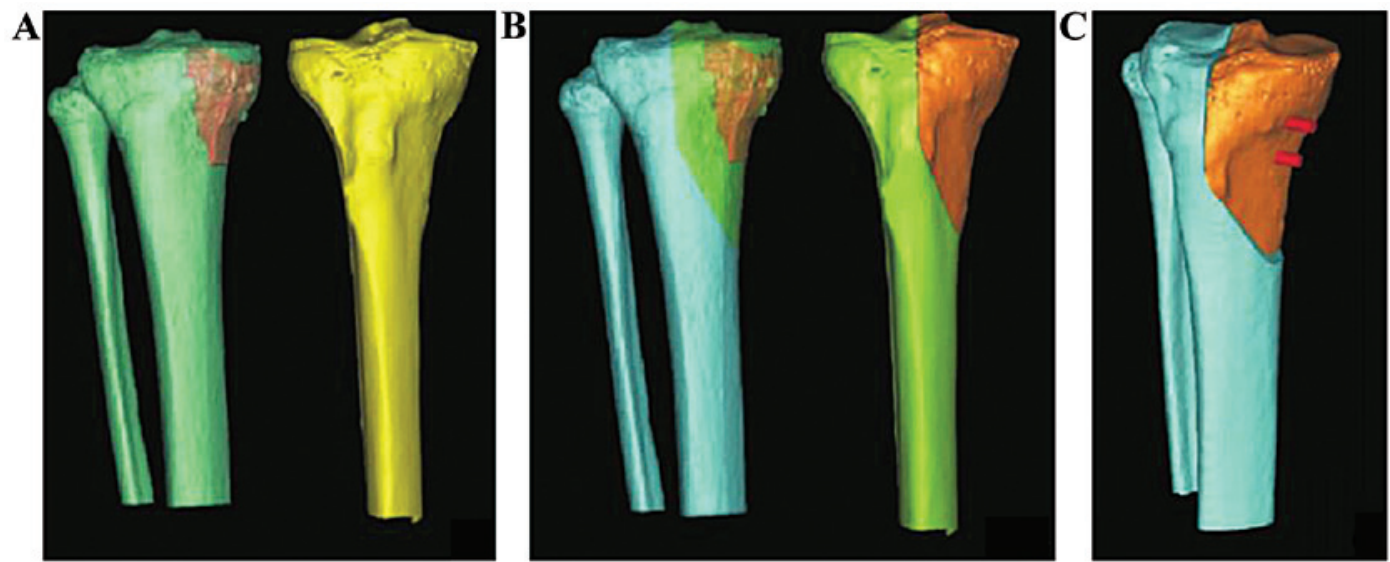

Figure 1. 3D modelling of osteotomy. (A) Tumor-tagged limb tibia and matched allografts, (B) projected osteotomy line and (C) simulated allogeneic bone pairing for internal fixation.

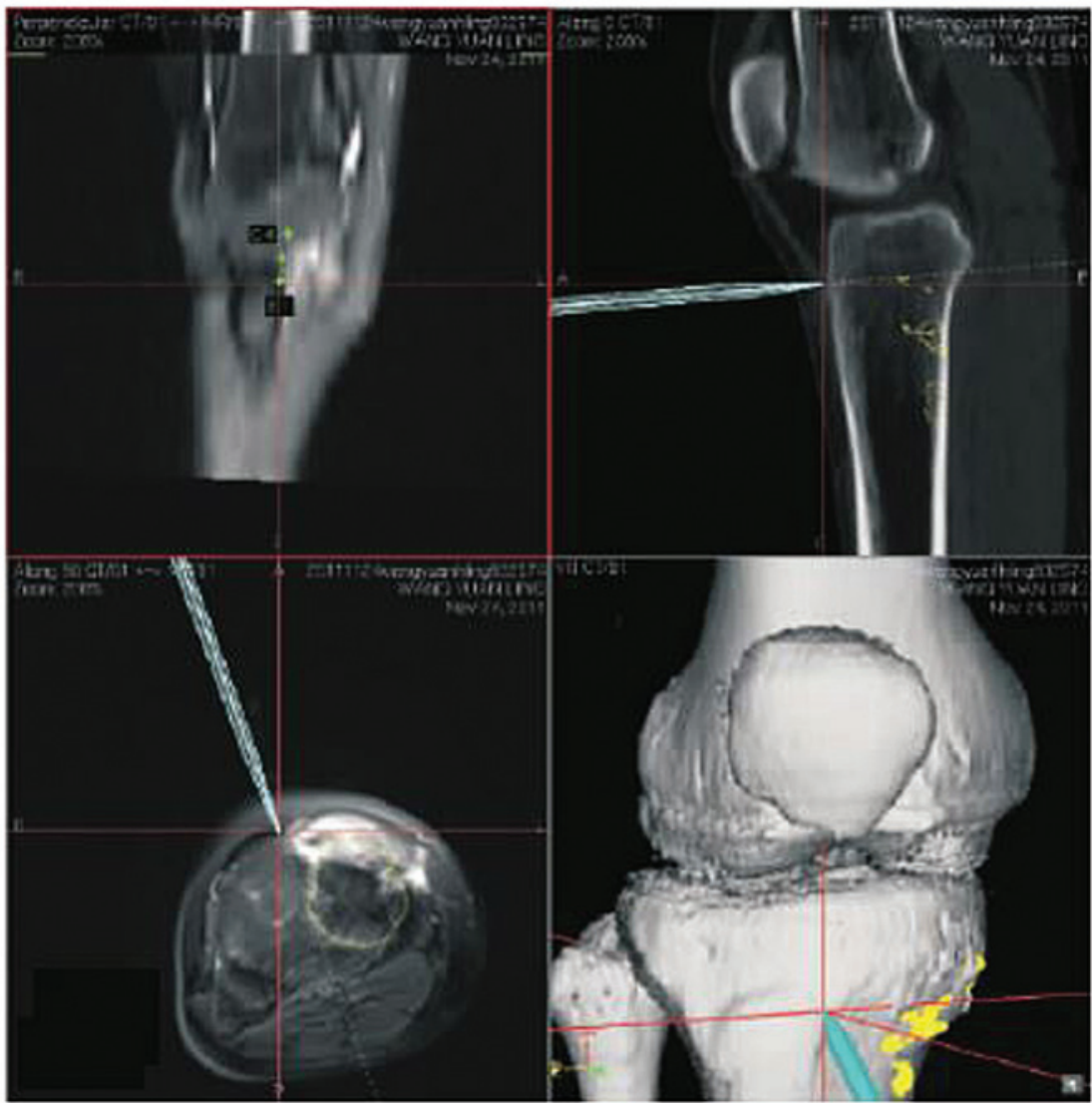

Figure 2. Image of the tumor osteotomy range marked on the 3D model of the upper tibia via the navigation system to guide the procedure.

tumor. According to pathology results 24 patients had osteosarcoma, 16 chondrosarcoma, and 5 Ewing's sarcoma. Finally, there were 20 patients with Enneking stage I and 25 with stage II; and the tumor diameter range was $0.5-15.5 \mathrm{~cm}$, with an average of $7.2 \pm 3.4 \mathrm{~cm}$.

Study methods. The same team participating in surgery and nursing of the patients completed this study. X-ray, CT and MRI examinations were performed before surgery. The original image data were saved as Dicom files, and analyzed with the Mimics 10.01 imported software (Materialise, Leuven, Belgium). Six CT tumor three-dimensional images were reconstructed and a precise osteotomy 3D solid model site was built up via image fusion technology (Fig. 1). The available bone marrow resources at the Xijing Hospital of the Fourth Military Medical University were recovered. Bone and cartilage tissues around the knee (such as upper and lower femur, and upper and lower tibia) were scanned via a 64-slice CT. Heterogeneity information such as the length, width, height and three-dimensional image of each specimen was stored in the Dicom format. At the same time, articular surface and specific bone markers were used to establish a digital bone bank. After that, tumor CT images were compared with the allogeneic bone and joint data to make the necessary 
Table I. Data of osteotomy range and allogeneic bone pairing comparisons.

\begin{tabular}{|c|c|c|c|c|c|c|c|}
\hline Group & Cases & $\begin{array}{l}\text { Tumor } \\
\text { resection } \\
\text { length }(\mathrm{cm})\end{array}$ & $\begin{array}{c}\text { Margin } \\
\text { negative rate } \\
\text { (cases, \%) }\end{array}$ & $\begin{array}{l}\text { Osteotomy } \\
\text { duration } \\
\text { (min) }\end{array}$ & $\begin{array}{l}\text { Pairing } \\
\text { time } \\
\text { (min) }\end{array}$ & $\begin{array}{l}\text { Registration } \\
\text { error } \\
(\mathrm{mm})\end{array}$ & $\begin{array}{l}\text { Pairing } \\
\text { error } \\
(\mathrm{mm})\end{array}$ \\
\hline Tumor recurrence & 5 & $11.6 \pm 3.5$ & $4(80.0)$ & $49.2 \pm 16.7$ & $34.6 \pm 12.3$ & $0.39 \pm 0.12$ & $0.12 \pm 0.05$ \\
\hline Tumor non-recurrence & 40 & $12.2 \pm 3.9$ & $38(95.0)$ & $45.6 \pm 18.2$ & $31.9 \pm 11.5$ & $0.34 \pm 0.11$ & $0.10 \pm 0.04$ \\
\hline$t / \chi^{2}$ & & 0.256 & - & 0.462 & 0.269 & 0.362 & 0.126 \\
\hline P-value & & 0.824 & 1.000 & 0.659 & 0.854 & 0.768 & 0.923 \\
\hline
\end{tabular}

measurements and to choose an optimal bone match. Mimics software version 16.0 (Media Cybernetics, Inc., Rockville, MD, USA) was used to simulate cleaning, compounding the lesion site, precisely calculating the range of tumor resection and allogeneic bone osteotomy.

Bone tumor and allogeneic bone image data were transmitted to the navigation system II-Cart (Stryker, Kalamazoo, MI, USA). Image fusion technique was used to construct the three-dimensional model of the surgical site and to mark the preoperative design of the osteotomy region on the 3D solid model (Fig. 2). The corresponding location and internal fixation position of allogeneic bone and joint were determined according to the osteotomy range. Three to five registration points were marked on the three-dimensional model based on the surgical approach and exposed range. A tracker was installed during surgery to reveal the registration points, which matched anatomical markers with corresponding sites on the three-dimensional model and guided the procedure. After the registration, the tumor was resected according to the preoperative plan, the allogeneic bone was prepared and the bone defect was filled based on the navigator's guidance, ensuring the pairing of osteotomy and articular surface. The location of internal fixation was determined via the navigation system instructions; lower limb line and joint angle were corrected until satisfactory.

Follow-up indicators. The success rate of osteotomy and pairing, average osteotomy time, pairing time, mean error of anatomical registration point, average error of tumor resection and allogeneic bone pairing were all calculated. Postoperative complications, tumor recurrence and survival rates, allogeneic bone healing time and knee joint functionality MSTS scores were evaluated after surgery.

Statistical analysis. The SPSS 20.0 statistical software (SPSS Inc., Chicago, IL, USA) was used for statistical analysis. Data were expressed as mean \pm standard deviation (SD), comparison between groups was tested via independent sample t-test, and results were expressed as number or percentages (\%). Comparison between groups was done via $\chi^{2}$ test or Fisher's exact test. $\mathrm{P}<0.05$ was considered to indicate a statistically significant diference.

\section{Results}

All patients underwent a successful osteotomy and allogeneic bone pairing. The osteotomy time ranged from 25 to $72 \mathrm{~min}$ with an average of $46.8 \pm 12.3 \mathrm{~min}$, and the pairing time ranged from 18 to $45 \mathrm{~min}$ with an average of $32.5 \pm 9.8 \mathrm{~min}$. The intraoperative anatomical registration points were satisfactorily paired with the virtual 3D CT images. The registration error ranged from 0.16 to $0.58 \mathrm{~mm}$, with an average of only $0.36 \pm 0.09 \mathrm{~mm}$. The tumor resection area and allogeneic osteoarticular osteotomy region were satisfactorily paired with an error ranging from 0.05 to $0.36 \mathrm{~mm}$ or $0.11 \pm 0.03 \mathrm{~mm}$ on average. There were no unequal limb or joint deformities after operation. The postoperative follow-up was carried out for 8.5 to 22.5 months, with a mean of 11.6 \pm 3.9 months. While 5 cases $(11.1 \%)$ had tumor recurrence, the survival rate during the follow-up period was $95.6 \%$ (43/45). There were no statistically significant differences in the length of resection, the size of incision margin, the osteotomy or matching durations or the registration or pairing errors between the allogeneic bone and the target defect $(\mathrm{p}>0.05)$ (Table I). Additionally, the healing time of allogeneic and host bones ranged from 3.6 to 8.2 months with an average of $5.5 \pm 1.2$ months, there were no flexible internal fixations, fractures or joint collapses. The knee joint functionality MSTS score ranged from 18.2 to 28.9 points (with an average of $25.5 \pm 6.6$ points) until the end of the follow-up period.

\section{Discussion}

The common complications of limb salvage surgery are prosthesis loosening, dislocation, allogeneic nonunion, rejection reaction, internal fixation fracture and infection (6). Allogeneic bone graft implantation increases the biomechanical strength compared with prosthesis (7), and also provides good articular surfaces and muscle, ligament and joint capsule attachment points (8). In general, a shorter time to bone healing means there is a higher probability of better stability and functionality of the joint (9). Many studies have proved that this method has better short-term and long-term clinical results and fewer complications $(10,11)$. Our study results are no exception.

The right selection of an allogeneic bone joint, especially the structure of the bone graft material is key to the success of the surgery. We took advantage of an established comprehensive digital bone bank library, combined with preoperative computer-simulated tumor resection and residual joint defect correction ranges, to choose the best pairing bone segment. The 3D information obtained by processing CT images of the bone defects aided in skeletal bone dressing and calculating the location and direction of internal fixation parameters. Intraoperative, real-time monitoring and the 
use of a computer-assisted navigation system allow surgeons to improve operation accuracy, accomplishing individual reconstruction requirements $(12,13)$. This study also analyzed data comparing parameters of tumor resection length, margin of incision, osteotomy and pairing durations, registration and pairing errors between allogeneic bone and bone defect in a group of patients who presented tumor recurrences and another that did not. The differences among groups were not statistically significant, suggesting that the computer-assisted navigation system for tumor resection, allogeneic bone pairing and joint reconstruction is not a technical factor affecting the tumor recurrence rate in the long run. The tumor biological characteristics and individual differences probably account for recurrence rate differences among patients (14), the specific mechanism remains to be analyzed.

China has established dozens of standardized bone banks, where the sources, preparation and preservation methods of allogeneic bones are guaranteed $(15,16)$. The technology of computer-assisted navigation has been well tested in neurosurgery, oral and maxillofacial deformity correction, artificial joint selection, prosthesis implantation and other medical practices $(17,18)$.

The evaluation of the cases reviewed in this study show that a computer-assisted navigation system for bone reconstruction after malignant bone tumor resection results in a high osteotomy accuracy and pairing success rate, achieves appropriate post-reconstruction posterior limb function levels, carries only a small risk of complications, and is therefore a safe and effective approach. Due to the small sample size and the less than desirable follow-up period of our study, further clinical studies are needed to validate our results.

\section{References}

1. Zhang P, Feng F, Cai Q, Yao W, Gao S, Wang J and Wang X: Effects of metaphyseal bone tumor removal with preservation of the epiphysis and knee arthroplasty. Exp Ther Med 8: 567-572, 2014.

2. Wong KC and Kumta SM: Joint-preserving tumor resection and reconstruction using image-guided computer navigation. Clin Orthop Relat Res 471: 762-773, 2013.

3. Rabitsch K, Maurer-Ertl W, Pirker-Frühauf U, Lovse T, Windhager $\mathrm{R}$ and Leithner A: Reconstruction of the distal radius following tumour resection using an osteoarticular allograft. Sarcoma 2013: 318767, 2013.
4. Wong KC and Kumta SM: Use of computer navigation in orthopedic oncology. Curr Surg Rep 2: 47, 2014.

5. Aponte-Tinao L, Ritacco LE, Ayerza MA, Muscolo DL, Albergo JI and Farfalli GL: Does intraoperative navigation assistance improve bone tumor resection and allograft reconstruction results? Clin Orthop Relat Res 473: 796-804, 2015.

6. Nakamura T, Matsumine A, Uchida A, Kawai A, Nishida Y, Kunisada T, Araki N, Sugiura H, Tomita M, Yokouchi M, et al: Clinical outcomes of kyocera modular limb salvage system after resection of bone sarcoma of the distal part of the femur: The Japanese Musculoskeletal Oncology Group Study. Int Orthop 38: 825-830, 2014.

7. Ogilvie CM, Crawford EA, Hosalkar HS, King JJ and Lackman RD: Long-term results for limb salvage with osteoarticular allograft reconstruction. Clin Orthop Relat Res 467: 2685-2690, 2009.

8. Fan H, Guo Z, Wang Z, Li J and Li X: Surgical technique: Unicondylar osteoallograft prosthesis composite in tumor limb salvage surgery. Clin Orthop Relat Res 470: 3577-3586, 2012.

9. Gharedaghi M, Peivandi MT, Mazloomi M, Shoorin HR, Hasani M, Seyf P and Khazaee F: Evaluation of clinical results and complications of structural allograft reconstruction after bone tumor surgery. Arch Bone Jt Surg 4: 236-242, 2016.

10. Aponte-Tinao LA, Ayerza MA, Muscolo DL and Farfalli GL: Allograft reconstruction for the treatment of musculoskeletal tumors of the upper extremity. Sarcoma 2013: 925413, 2013.

11. Teunis T, Nota SP, Hornicek FJ, Schwab JH and LozanoCalderón SA: Outcome after reconstruction of the proximal humerus for tumor resection: A systematic review. Clin Orthop Relat Res 472: 2245-2253, 2014.

12. Wong KC and Kumta SM: Computer-assisted tumor surgery in malignant bone tumors. Clin Orthop Relat Res 471: 750-761, 2013 .

13. Meijer MF, Stevens M, Boerboom AL, Bulstra SK and Reininga IH: The influence of computer-assisted surgery on rotational, coronal and sagittal alignment in revision total knee arthroplasty. BMC Musculoskelet Disord 15: 94, 2014.

14. Ding HW, Yu GW, Tu Q, Liu B, Shen JJ, Wang H and Wang YJ: Computer-aided resection and endoprosthesis design for the management of malignant bone tumors around the knee: Outcomes of 12 cases. BMC Musculoskelet Disord 14: 33, 2013.

15. Wang W, Bi WZ, Yang J, Han G and Jia JP: Pelvic reconstruction with allogeneic bone graft after tumor resection. Acta Ortop Bras 21: 150-154, 2013.

16. Cheng B, Lu SL and Fu XB: Regenerative medicine in China: Main progress in different fields. Mil Med Res 3: 24, 2016.

17. Ort R, Metzler P, Kruse AL, Matthews F, Zemann W, Grätz KW and Luebbers HT: The reliability of a three-dimensional photo system (3dMDface) based evaluation of the face in cleft lip infants. Plast Surg Int 2012: 138090, 2012.

18. Al Eissa S, Al-Habib AF and Jahangiri FR: Computer-assisted navigation during an anterior-posterior en bloc resection of a sacral tumor. Cureus 7: e373, 2015. 